\title{
Temperature and Chemical Reaction Distribution of a Laminar Diffusion Flame Measured by X-ray Compton Scattering
}

\author{
Hiroshi Sakurai ${ }^{1, *\left(\mathbb{D}, \text { Naruki Tsuji }^{2} \text {, Yoshio Zama }\right.}{ }^{1}$, Kosuke Suzuki ${ }^{1}{ }^{\mathbb{D}}$, Kazushi Hoshi $^{1}$, Daisuke Hiramoto ${ }^{1}$, \\ Yoshiharu Sakurai ${ }^{2}$ and Tomohiko Furuhata ${ }^{1}$ \\ 1 School of Science and Technology, Gunma University, Gunma 376-8515, Japan; yzama@gunma-u.ac.jp (Y.Z.); \\ kosuzuki@gunma-u.ac.jp (K.S.); hoshi@gunma-u.ac.jp (K.H.); t181d061@gunma-u.ac.jp (D.H.); \\ tfuruhata@gunma-u.ac.jp (T.F.) \\ 2 Japan Synchrotron Radiation Research Institute, Hyogo 679-5198, Japan; ntsuji@spring8.or.jp (N.T.); \\ sakurai@spring8.or.jp (Y.S.) \\ * Correspondence: sakuraih@gunma-u.ac.jp
}

check for

updates

Citation: Sakurai, H.; Tsuji, N.;

Zama, Y.; Suzuki, K.; Hoshi, K.;

Hiramoto, D.; Sakurai, Y.; Furuhata, T.

Temperature and Chemical Reaction

Distribution of a Laminar Diffusion

Flame Measured by X-ray Compton

Scattering. Crystals 2021, 11, 787.

https://doi.org/10.3390/

cryst11070787

Academic Editor: Benoit Heinrich

Received: 28 May 2021

Accepted: 2 July 2021

Published: 6 July 2021

Publisher's Note: MDPI stays neutral with regard to jurisdictional claims in published maps and institutional affiliations.

Copyright: (c) 2021 by the authors. Licensee MDPI, Basel, Switzerland. This article is an open access article distributed under the terms and conditions of the Creative Commons Attribution (CC BY) license (https:// creativecommons.org/licenses/by/ $4.0 /)$

\begin{abstract}
A laminar diffusion flame was measured by X-ray Compton scattering. The temperature distribution was measured from an analysis of Compton scattered X-ray intensity. The chemical state distribution was obtained from a Compton scattered X-ray spectrum analysis (s-parameter analysis). The analysis of intensity and s-parameter of Compton scattered X-ray spectra indicate that the propane molecule emitted from the cylindrical Bunsen burner collapse immediately coincides with soot generation. Furthermore, the temperature increases up to $1500 \mathrm{~K}$ and a large amount of $\mathrm{CO}_{2}$ was generated at the combustion field. Our results show that the Compton scattered X-ray analysis can be a novel nondestructive measurement for temperature and chemical states in a combustion reaction.
\end{abstract}

Keywords: laminar diffusion flame; Compton scattering; temperature distribution; soot

\section{Introduction}

Conventional fossil fuels still occupy more than $90 \%$ of global energy consumption although there are many alternative methods to replace fossil fuels by renewable energy such as solar power. The main drawback of the usage of fossil fuels is that the combustion process emits pollutants such as soot [1]. People have paid more attention to air quality, which has become one of the main factors for human health. Reduction in pollutant emission in fossil fuels combustion is urgently needed. Therefore, the study of the combustion process is important.

Since combustion occurs due to complex interactions and heat, mass, and momentum transfer, measurements of distributions for temperature and chemical states are a key consequence for the elucidation of combustion phenomena. Nonintrusive measurements offer important advantages to study combusting process since they do not insert a disturbance that can influence flow characteristics and affect the temperature distribution [2].

Recently, we suggested a temperature measurement for a laminar premixed flame by measuring a Compton scattered X-ray intensity, which can be a nonintrusive measurement of the temperature distribution for combustion gas in a flame [3]. Furthermore, we demonstrated a possibility to probe chemical states from an analysis of energy spectrum of Compton scattered X-rays because the energy spectrum of Compton scattered X-rays corresponds to an electron momentum distribution of a material, which reflects wavefunctions (in other words, chemical states) in the momentum space [4]. Therefore, the Compton scattered X-ray measurement can be a nondestructive measurement for temperature and chemical states in a combustion reaction, which elucidates combustion phenomena for the reduction pollutant emissions in fossil fuels combustion.

In this study we report a measurement of a laminar diffusion flame probed by Compton scattered X-rays. The distributions of temperature and chemical states are discussed from a viewpoint of combustion reactions in the flame. 


\section{Flame Measurement by Compton Scattered X-rays}

A Compton-scattered X-ray spectrum provides an electron momentum distribution, which is called Compton profile, $J\left(p_{z}\right)$, under the impulse approximation described as follows.

$$
\begin{gathered}
J\left(p_{z}\right)=\iint n(\mathbf{p}) \mathrm{d} p_{x} \mathrm{~d} p_{y}, \\
n(\mathbf{p})=\sum_{i}^{o c c}\left|\chi_{i}(\mathbf{p})\right|^{2}, \\
\chi_{i}(\mathbf{p})=\left(\frac{1}{\sqrt{2 \pi \hbar}}\right)^{3} \int \psi_{i}(\mathbf{r}) \exp \left(-\frac{i \mathbf{p r}}{\hbar}\right) d \mathbf{r} . \\
\frac{p_{z}}{m c}=\frac{E_{2}-E_{1}+\left(E_{2} E_{1}(1-\cos \theta) / m c^{2}\right)}{\sqrt{E_{1}^{2}+E_{2}^{2}-2 E_{2} E_{1} \cos \theta}}
\end{gathered}
$$

Here, $\mathbf{p}\left(=\left(p_{x}, p_{y}, p_{z}\right)\right)$ denotes the momentum of an electron in a target material. $E_{1}$ and $E_{2}$ denote an incident and scattered X-ray energy with a scattering angle $\theta$, respectively. The electron mass and the velocity of light are denoted by $m$ and $c$. Since the molecules are oriented randomly in the space, we can regard $p_{z}=p_{x}=p_{y}$. An electron momentum density is denoted by $n(\mathbf{p}) . \psi_{i}(\mathrm{r})$ and $\chi_{i}(\mathbf{p})$ denote the wavefunction of the $i$-th electronic state in real space and momentum space, respectively. Therefore, we can obtain information on a wavefunction, in other words, measurements on the chemical states from a Compton profile (electron momentum distribution) [4].

The intensity of Compton-scattered X-rays for monochromatic X-ray beams, $\mathrm{dN}$, is expressed by the following:

$$
\mathrm{d} N=\Phi_{0} t_{1} t_{2} \rho_{e} \mathrm{~d} V \frac{\mathrm{d} \sigma_{\mathrm{KN}}}{\mathrm{d} \Omega}
$$

where $\Phi_{0}$ denotes the incident photon flux into a target object, $t_{1}$ denotes the incident $\mathrm{X}$-ray transmittance to a probing volume in the object, $t_{2}$ denotes the scattered $X$-ray transmittance from the probing volume to an X-ray detector, $\rho_{e}$ denotes the average electron density over the probing volume $\mathrm{d} V$, and $\mathrm{d} \sigma_{\mathrm{KN}} / \mathrm{d} \Omega$ denotes the Klein-Nishina differential cross section [5]. In the case of light element gases, $t_{1}$ and $t_{2}$ can be regarded as constant values. The intensity of Compton scattered X-rays, $\mathrm{dN}$, corresponds to the electron density $\rho_{e}$. A ratio of a molecular density to an electron density, $\alpha$, is defined by the following equation.

$$
\alpha=\rho_{m} / \rho_{e}
$$

Here, $\rho_{m}$ is a molecular density. Since the flame system can be regarded as the ideal gas law [6], the temperature distribution is obtained from the electron density as described as follows.

$$
\rho_{m} T=\alpha \rho_{e} T=\frac{P}{R}
$$

Here, $\rho_{m}=n / V$ denotes the molecular density while $T$ and $R$ denotes the temperature and the ideal gas constant, respectively. The ratio of the molecular density to the electron density, $\alpha$, is obtained by assuming a combustion reaction. Here, we regard $\alpha$ as a constant value of $\alpha_{\text {air }}=0.0693$ within an error of $2 \%$ in the combustion reaction [3]. The ambient pressure, $P$, is regarded as a constant. The ambient temperature of the air is $298 \mathrm{~K}$. Therefore, we can estimate the temperature distribution from the Equation (7).

\section{Materials and Methods}

The experiment was performed at the BL08W beamline [7] in SPring- 8 . The linearly polarized X-rays were obtained from an elliptical multipole wiggler [8]. The synchrotron radiations were monochromatized and horizontally focused by an asymmetric Johann-type Si monochromator with (620) reflecting planes [9] to deliver $115.56 \mathrm{keV} X$-ray beams, which are the optimized energy on BL08W. Figure 1a shows a picture of the experimental setup 
of the present experiment. The size of the incident X-rays was defined by the entrance slit system to be $1 \mathrm{~mm}$ in height and $1 \mathrm{~mm}$ in width. An energy spectrum of Compton scattered X-rays from a flame in a probing volume was detected by a Ge solid state detector (Ge-SSD) with a collimating silt of $1 \mathrm{~mm}$ in height and $1 \mathrm{~mm}$ in width at a scattering angle of 133.8 degrees. Therefore, the probing volume was about $1 \mathrm{~mm}^{3}$. The intensity of incident $\mathrm{X}$-ray beams was monitored by an ion-chamber for data normalization. Compton scattered $\mathrm{X}$-rays were measured for $60 \mathrm{~s}$ with a $2 \mathrm{~mm}$ step in the $x$-axis and $5 \mathrm{~mm}$ step in the $z$-axis. A Compton scattered X-ray spectrum was measured for $38 \mathrm{~min}$. A cylindrical Bunsen burner (inner diameter, $d_{\text {in }}=10 \mathrm{~mm}$ ) was used to provide pure propane ( $99 \mathrm{wt} . \%, 60 \mathrm{~mL} / \mathrm{min}$ ). The burner provided a laminar diffusion flame as shown in Figure 1b. The burner was mounted on a movable stage and the intensity of Compton scattered X-rays from the probing volume was measured by scanning with the X-ray beams along the vertical $(z)$ direction and horizontal $(x)$ direction, which were on a plane perpendicular to the incident $\mathrm{X}$-ray beam direction.

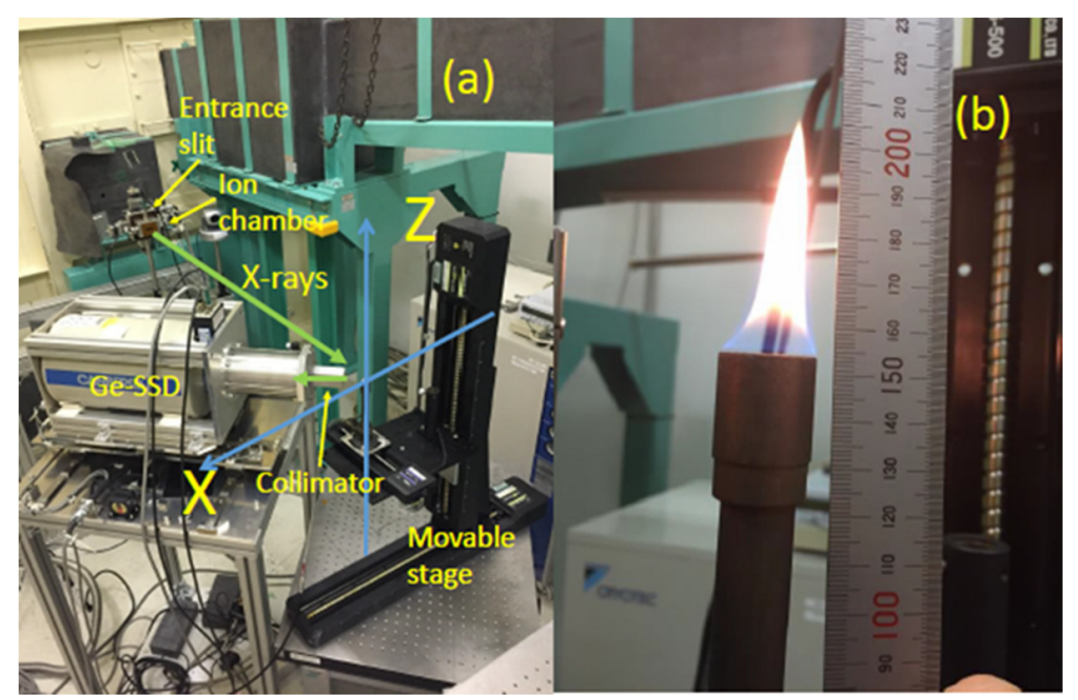

Figure 1. (a) Photograph of experimental setup of the present experiment. (b) Photograph of the present laminar diffusion flame.

\section{Results and Discussions}

Figure $1 \mathrm{~b}$ shows the side-view photograph of the laminar diffusion flame as explained previously. The flame shows an asymmetrical shape for the cylindrical axis of the burner. This comes from a self-induced air flow by the asymmetrical setup of the experimental system. The bright light emission of the flame corresponds to a thermal radiation of soot particles with a high temperature.

Figure 2a shows a cross sectional map of Compton scattered X-ray intensities for the flame shown in Figure 1b. The intensities are normalized to those of the air. The red region shows strong $\mathrm{X}$-ray intensities and the blue region shows weak intensities. The line of $x=-4 \mathrm{~mm}$ corresponds to the cylindrical axis of the burner. The relative intensity shows an asymmetrical shape for the cylindrical axis of the burner, which reflects the asymmetrical shape of the flame shown in Figure $1 b$.

Figure $2 \mathrm{~b}$ shows Compton scattered X-ray spectra from positions of $(x, z)=(-4 \mathrm{~mm}$, $2.5 \mathrm{~mm}),(-4 \mathrm{~mm}, 15 \mathrm{~mm}),(-4 \mathrm{~mm}, 30 \mathrm{~mm}),(-4 \mathrm{~mm}, 50 \mathrm{~mm})$, and $(12 \mathrm{~mm}, 60 \mathrm{~mm})$, which are shown as dots in Figure 2a. The position of $(x, z)=(12 \mathrm{~mm}, 60 \mathrm{~mm})$ corresponds to the ambient atmosphere of air. The position of $(x, z)=(-4 \mathrm{~mm}, 2.5 \mathrm{~mm})$ corresponds to pure propane. The intensities remarkably decrease at $\mathrm{z}=15 \mathrm{~mm}$ and $30 \mathrm{~mm}$ compared to that of air, which reflects a high temperature region of the flame. The Compton scattered X-ray spectra can be converted into electron momentum distributions, $J\left(p_{z}\right)$, as shown in Figure 2c. Since Compton profiles probe the wavefunctions in the momentum space 
as shown in Equations (1)-(3), shapes of Compton profiles reflect the chemical states as explained previously.
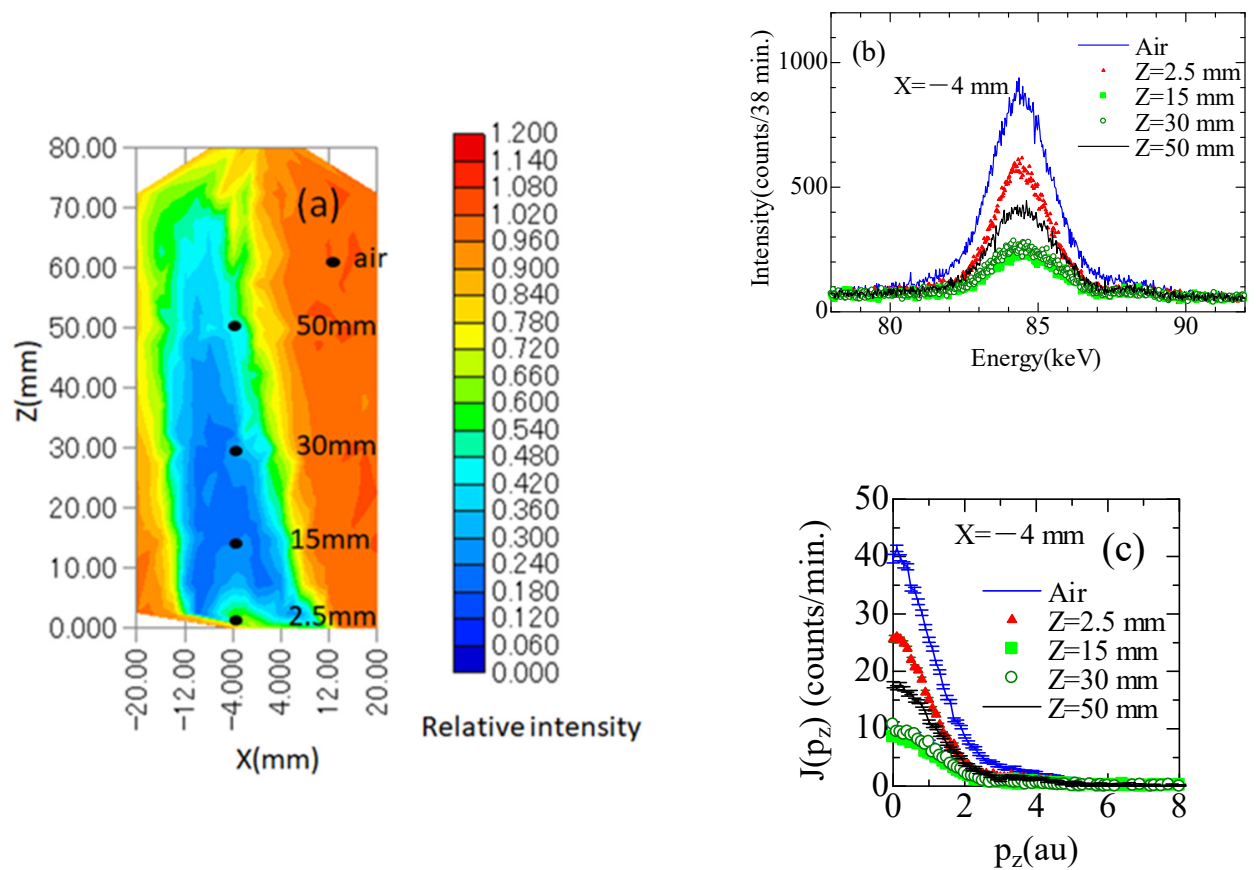

Figure 2. (a) Compton scattering X-ray intensity distribution of a laminar diffusion flame shown in Figure $1 \mathbf{b}$. The intensities are normalized those of air. (b) Compton scattered X-ray spectra at $z=2.5 \mathrm{~mm}, 15 \mathrm{~mm}, 30 \mathrm{~mm}$, and $50 \mathrm{~mm}$ along $x=-4 \mathrm{~mm}$ and air shown in (a). (c) Compton profiles (electron momentum distributions) obtained from (b).

In order to highlight the change of the shape in the Compton profiles, we obtain a difference of Compton profiles as described as follows.

$$
\begin{aligned}
J\left(p_{z}\right) & =J\left(p_{z}\right) / \int J\left(p_{z}\right) d p_{z} \\
\Delta J^{\prime}\left(p_{z}\right) & =J^{\prime}{ }_{z m m}\left(p_{z}\right)-J^{\prime}{ }_{\text {air }}\left(p_{z}\right)
\end{aligned}
$$

Here a Compton profile, $J\left(p_{z}\right)$, shown in Figure $2 \mathrm{c}$ is normalized into $J^{\prime}\left(p_{z}\right)$, which has a unit area as Equation (8). $J^{\prime} z m m\left(p_{z}\right)$ denotes a normalized Compton profile at $z \mathrm{~mm}$. $J^{\prime}$ air $\left(p_{z}\right)$ denotes the normalized Compton profile of air.

Differences of Compton profiles, $\Delta J\left(p_{z}\right)$, in Equation (9) are shown in Figure 3. The difference Compton profile between the propane and air $(z=2.5 \mathrm{~mm})$ shows positive value around $p_{z}=0$ au. This indicates that the Compton profile of the propane has a rather acute shape than compared to air. The difference in Compton profiles at $z=15 \mathrm{~mm}$ and $30 \mathrm{~mm}$ show negative values around $p_{z}=0 \mathrm{au}$, which indicates that the Compton profiles at $z=15 \mathrm{~mm}$ and $30 \mathrm{~mm}$ have rather obtuse shape than compared to air.

This situation can be understood from theoretical calculations of Compton profiles relative to air, propane, $\mathrm{H}_{2} \mathrm{O}$, and $\mathrm{CO}_{2}$ by Crystal14 code [10], as shown in Figure 4, in which the Kohn-Sham DFT Hamiltonian with the basis function of the STO-3G was used. Here, molecular directions are spherically averaged. The calculated Compton profiles are normalized to have a unit area. The Compton profiles of propane and $\mathrm{CO}_{2}$ have rather acute and obtuse shapes, respectively, than compared with air, which are consistent with Figure 3. This indicates that the obtuse shapes of the Compton profiles at $z=15 \mathrm{~mm}$ and $30 \mathrm{~mm}$ are ascribed to the feature of the Compton profile for $\mathrm{CO}_{2}$ molecules. 


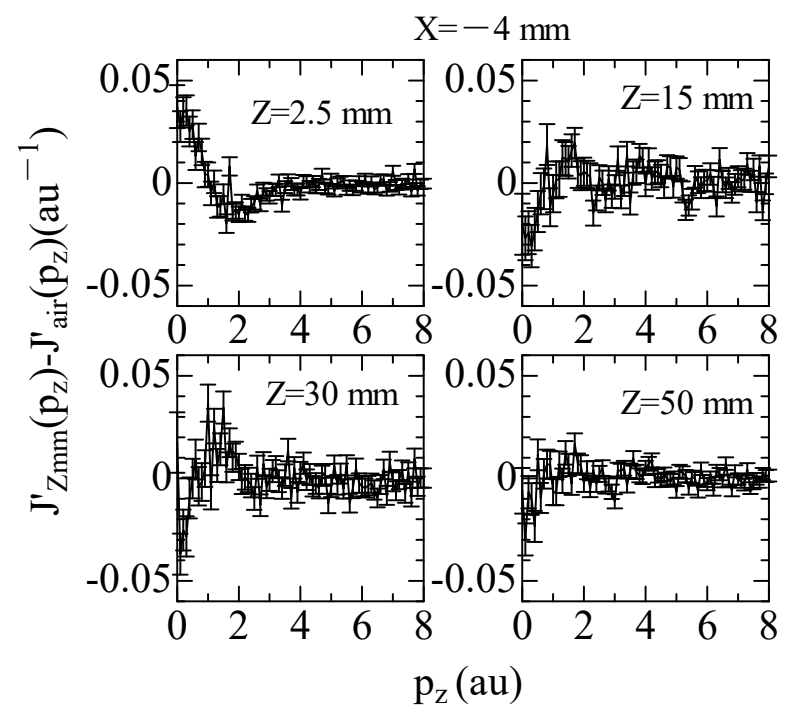

Figure 3. The difference of Compton profiles $\Delta J\left(p_{z}\right)=J^{\prime}{ }_{z m m}\left(p_{z}\right)-J_{\text {air }}^{\prime}\left(p_{z}\right)$. Here, $J_{z m m}^{\prime}\left(p_{z}\right)$ denotes a normalized Compton profile $J^{\prime}\left(p_{z}\right)$ at $z \mathrm{~mm}$. $J^{\prime}$ air $\left(p_{z}\right)$ denotes the normalized Compton profile of air.

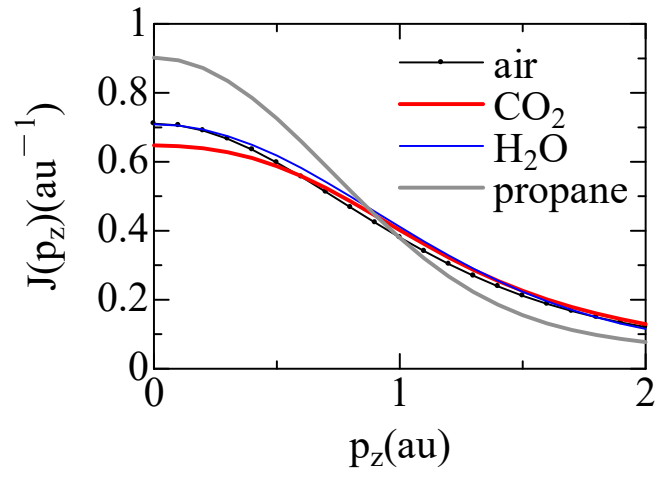

Figure 4. Theoretical Compton profiles of propane, $\mathrm{CO}_{2}, \mathrm{H}_{2} \mathrm{O}$, and air by crystal code, in which the molecular axis is spherically averaged. The Compton profiles are normalized to have a unit area.

If we assume the system as an ideal gas [6], we can estimate the temperature of the flame from Equation (7). Figure 5a shows the estimated temperature of the flame. Here the air, shown as region $e$, is regarded to have the average temperature of $298 \mathrm{~K}$. The estimated temperature in region $a$ is about $500 \mathrm{~K}$, which includes $z=2.5 \mathrm{~mm}$ and is dominated by propane molecules just prior to the combustion. The region $b$ has the temperature of approximately $1000 \mathrm{~K}$ and corresponds to the transition area of the combustion reaction from propane to $\mathrm{CO}_{2}$ in which soot generation was reported [11,12]. The highest temperature region (shown as the region $c$ ), which includes $z=15 \mathrm{~mm}$ and $30 \mathrm{~mm}$, shows about $1500 \mathrm{~K}$. The region $d$, which includes $z=50 \mathrm{~mm}$, corresponds to the mixing region of the high temperature combustion gas and air and the temperature decreases as $z$ increases. These results indicate that the temperature distribution should have a relation to the distribution of chemical states as discussed in Figure 3.

In order to obtain the distribution of the chemical state, we analyzed the shape of the Compton profile by the s-parameter analysis [13,14]. The cross section of Compton scattering is dominated by the electron density as shown in Equation (5). Therefore, it is not easy to distinguish the elements from the Compton scattering intensities. However, an electron momentum distribution is sensitive to the chemical states, including the element composition. A shape analysis of the Compton scattered X-ray spectrum is useful. We confirmed the linearity of the s-parameter against the Li concentration and succeeded in obtaining in operando Li concentrations in a Li ion battery [13,14]. The s-parameter can be sensitive to the chemical states of the combustion reaction. Therefore, we tried the s-parameter analysis as the first step. 

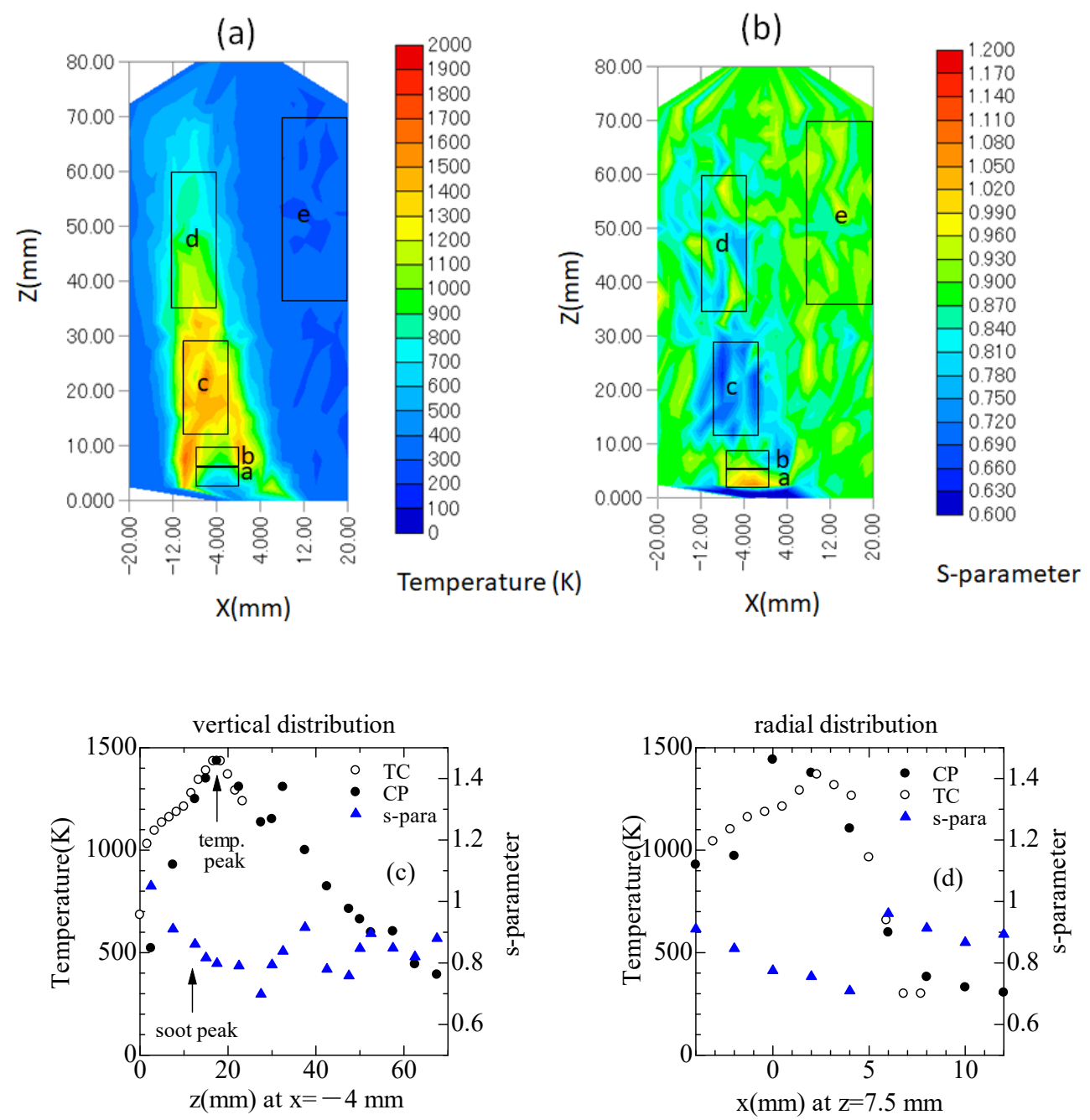

Figure 5. (a) Temperature distribution of the laminar diffusion flame obtained from Figure $2 \mathrm{a}, \mathrm{b}$ s-parameter distribution of the laminar diffusion flame. (c) The temperature distributions along $z$-axis at $x=-4 \mathrm{~mm}$ in $(\mathbf{a}, \mathbf{b})$ measured by Compton scattering (CP) and a thermocouple (TC). The s-parameters (s-para) are also shown. (d) The temperatures (CP and TC) and s-parameter (s-para) distributions along to the radial direction ( $x$-axis).

The s-parameter is defined as the follows.

$$
s=\frac{S_{L}}{S_{H}}=\frac{\int_{-d}^{d} J\left(p_{z}\right) d p_{z}}{\int_{-r}^{-d} J\left(p_{z}\right) d p_{z}+\int_{d}^{r} J\left(p_{z}\right) d p_{z}}
$$

Here, $S_{L}$ and $S_{H}$ are the areas of a Compton profile, which correspond to the lowmomentum and high-momentum regions. The low and high momentum regions are defined as $\left|p_{z}\right| \leq d$ and $d \leq\left|p_{z}\right| \leq r$, respectively. In this study, $d=1$ atomic units (au) and $r=5$ au are used. The acute shape provides a higher s-parameter and the obtuse shape provides a lower s-parameter. Figure $5 \mathrm{~b}$ shows the s-parameter distribution of the flame. The highest s-parameter values are observed in region $a$, which corresponds to the acute shape of the Compton profile for propane molecules, as discussed in Figure $3(z=2.5 \mathrm{~mm})$. The s-parameter becomes lower value in region $b$, which corresponds to the collapse of propane molecules coinciding with the soot generation. The s-parameters are the lowest in region $c$, which correspond to the obtuse shape of the Compton profile for $\mathrm{CO}_{2}$, as discussed in Figure $3(z=15 \mathrm{~mm}$ and $30 \mathrm{~mm})$. The s-parameter converges to the value of air in region $d$. Figure $5 \mathrm{c}$ shows a comparison of measured temperatures between the present Compton scattering and a thermocouple along the $z$ direction at $x=-4 \mathrm{~mm}$ (vertical distribution). The 
s-parameter distribution is also shown. The measured temperatures by Compton scattering almost agree with those measured by the thermocouple. The tendency of the temperature distributions in Figure $5 \mathrm{c}$ agrees with recent simulated temperature distributions in a laminar diffusion flame [1,15]. The highest s-parameter values of 1.05 is observed at $z=2.5 \mathrm{~mm}$, which corresponds to propane molecules just before combustion around $500 \mathrm{~K}$, as shown in Figure $5 \mathrm{a}, \mathrm{b}$. The s-parameter value is about 0.85 and the temperature is about $1000 \mathrm{~K}$ at $z=12 \mathrm{~mm}$, which corresponds to the collapse of propane molecules coinciding with the soot generation maximum $[12,13]$. The lowest s-parameter value of 0.8 and the highest temperature around $1500 \mathrm{~K}$ are observed at $z=18 \mathrm{~mm}$, which indicates the amount of $\mathrm{CO}_{2}$ generation, as discussed before. Figure $5 \mathrm{~d}$ shows a comparison of measured temperatures between the present Compton scattering $(z=7.5 \mathrm{~mm})$ and a thermocouple $(z=6 \mathrm{~mm})$ along the radial direction ( $x$ direction). The s-parameter distribution is also shown. The measured temperatures almost agree between the Compton scattering and the thermocouple. The tendency of the radial distributions on the temperature shown in Figure $5 \mathrm{~d}$ agrees with recent reports $[15,16]$. The maximum temperature about $1500 \mathrm{~K}$ is observed around $x=0-2 \mathrm{~mm}$ with the lowest s-parameter values corresponding to the amount of $\mathrm{CO}_{2}$ generation. The flame front is observed around $x=5 \mathrm{~mm}$, where the s-parameter increases suddenly.

By comparing Figure 5 a to Figure $5 b$, a combustion process can be comprehended.

Figure 6 shows a relation of temperature in Figure 5a and s-parameter in Figure $5 b$. The propane molecules just prior to the combustion in region $a$ (temperature $500 \mathrm{~K}$ ) induces combustion reactions and immediately collapses in region $b$, coinciding with soot generation (temperature $1000 \mathrm{~K}$ ). The temperature increases up to $1500 \mathrm{~K}$ and s-parameter decreases in region $c$. The decrease in the s-parameter corresponds to the obtuse shape of Compton profiles, which reflects a large amount of $\mathrm{CO}_{2}$ generation. Afterwards, the combustion gas mix with air in region $d$. The temperature decreases and s-parameter converges relative to the value of the air (region $e$ ).

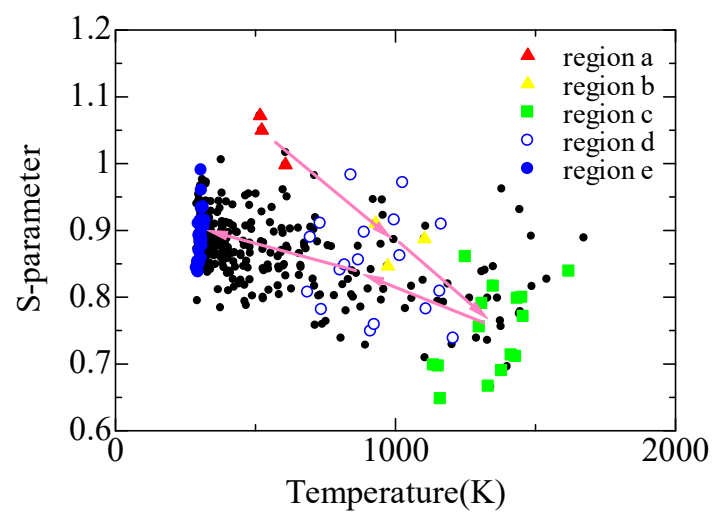

Figure 6. Temperatures and s-parameters of the laminar diffusion flame shown in Figure 5a,b. Propane molecules in region $a$ collapses, which immediately coincides with soot generation in region $b$. The temperature increases up to $1500 \mathrm{~K}$ and s-parameter decreases in region $c$ where the combustion filed is dominated by $\mathrm{CO}_{2}$ generation. Afterwards, the combustion gas mixes with air in region $d$. The temperature decreases and s-parameter converges to the value of the air (region $e$ ).

\section{Conclusions}

A laminar diffusion flame was measured by X-ray Compton scattering. The temperature distribution was measured from the analysis of Compton scattering intensity. The chemical state distribution was obtained by s-parameter analysis. The propane molecule emitted from the cylindrical Bunsen burner collapse immediately coincides with soot generation at about $1000 \mathrm{~K}$. The temperature increases up to $1500 \mathrm{~K}$ and large amounts of $\mathrm{CO}_{2}$ are generated at the combustion field. These results demonstrate that high-energy $\mathrm{X}$-ray Compton scattering can be employed as an in-situ technique to probe the inside of a combustion reaction. This technique will open up to the further applications for the 
elucidation of the combustion reaction for different gases, such as $\mathrm{CH}_{4}, \mathrm{NH}_{3}$, and $\mathrm{H}_{2}$, which reduces the emission of $\mathrm{CO}_{2}$.

Author Contributions: Writing—original draft preparation, H.S.; writing—review and editing, Y.Z., K.H. and Y.S.; experimental design, N.T.; theoretical calculations, K.S.; data analysis, D.H.; project administration, H.S. and T.F.; funding acquisition, H.S. All authors have read and agreed to the published version of the manuscript.

Funding: This work was supported by JSPS KAKENHI Grant Number 19K04464.

Institutional Review Board Statement: Not applicable.

Informed Consent Statement: Not applicable.

Data Availability Statement: The data presented in this study are available on request from the corresponding author.

Acknowledgments: These experiments were performed with the approval of the Japan Synchrotron Research Institute (JASRI) (Proposal No. 2017B1506) and the Human Resource Cultivation Center (HRCC), Gunma University (Proposal 2017-2020). This work was supported by JSPS KAKENHI 19K04464 (Grant-in-aid for Scientific Research (C)).

Conflicts of Interest: The authors declare no conflict of interest.

\section{References}

1. Lee, C.C.; Tran, M.-V.; Scribano, G.; Chong, C.T.; Ooi, J.B.; Cong, H.T. Numerical Study of NOx and Soot Formations in Hydrocarbon Diffusion Flames. Energy Fuels 2019, 33, 12839-12851. [CrossRef]

2. Xiao, X.; Choi, C.W.; Puri, I.K. Temperature measurements in steady two-dimensional partially premixed flames using laser interferometric holography. Combust. Flame 2000, 120, 318-332. [CrossRef]

3. Sakurai, H.; Kawahara, N.; Itou, M.; Tomita, E.; Suzuki, K.; Sakurai, Y. Densitometry and temperature measurement of combustion gas by X-ray Compton scattering. J. Synchrotron Radiat. 2016, 23, 617-621. [CrossRef] [PubMed]

4. Cooper, M.J. Compton scattering and electron momentum determination. Rep. Prog. Phys. 1985, 48, 415-481. [CrossRef]

5. Sharaf, J. Practical aspects of Compton scatter densitometry. Appl. Radiat. Isot. 2001, 54, 801-809. [CrossRef]

6. Tieng, S.M.; Lai, W.Z.; Fujiwara, T. Holographic temperature measurement on axisymmetric propane-air, fuel-lean flame. Meas. Sci. Technol. 1992, 3, 1179-1187. [CrossRef]

7. Sakurai, Y. High-Energy Inelastic-Scattering Beamline for Electron Momentum Density Study. J. Synchrotron Radiat. 1998, 5, 208-214. [CrossRef] [PubMed]

8. Maréchal, X.-M.; Hara, T.; Tanabe, T.; Tanaka, T.; Kitamura, H. Development of an elliptical multipole wiggler at SPring-8. J. Synchrotron Radiat. 1998, 5, 431-433. [CrossRef] [PubMed]

9. Yamaoka, H.; Hiraoka, N.; Ito, M.; Mizumaki, M.; Sakurai, Y.; Kakutani, Y.; Koizumi, A.; Sakai, N.; Higashi, Y. Performance of bentcrystal monochromators for high-energy synchrotron radiation. J. Synchrotron Radiat. 2000, 7, 69-77. [CrossRef] [PubMed]

10. Dovesi, R.; Orlando, R.; Erba, A.; Zicovich-Wilson, C.; Civalleri, B.; Casassa, S.; Maschio, L.; Ferrabone, M.; De La Pierre, M.; D'Arco, P.; et al. CRYSTAL14: A program for the ab initio investigation of crystalline solids. Int. J. Quantum Chem. 2014, 114, 1287-1317. [CrossRef]

11. Zama, Y.; Shimizu, K.; Tsukui, M.; Furuhata, T.; Arai, M. Relationship between O2 Concentration and Soot Formation in Propane Laminar Diffusion Flame. Trans. Jpn. Soc. Mech. Eng. Ser. B 2013, 79, 1147-1154. [CrossRef]

12. Kobashi, Y.; Zama, Y.; Kuboyama, T. Modeling wall film formation and vaporization of a gasoline surrogate fuel. Int. J. Heat Mass Transf. 2020, 147, 119035. [CrossRef]

13. Suzuki, K.; Barbiellini, B.; Orikasa, Y.; Kaprzyk, S.; Itou, M.; Yamamoto, K.; Wang, Y.J.; Hafiz, H.; Uchimoto, Y.; Bansil, A.; et al. Non-destructive measurement of in-operando lithium concentration in batteries via x-ray Compton scattering. J. Appl. Phys. 2016, 119, 025103. [CrossRef]

14. Suzuki, K.; Suzuki, A.; Ishikawa, T.; Itou, M.; Yamashige, H.; Orikasa, Y.; Uchimoto, Y.; Sakurai, Y.; Sakurai, H. In operando quantitation of Li concentration for a commercial Li-ion rechargeable battery using high-energy X-ray Compton scattering. J. Synchrotron Radiat. 2017, 24, 1006-1011. [CrossRef] [PubMed]

15. Chu, H.; Yan, Y.; Xiang, L.; Han, W.; Ren, F.; Peng, L. Effect of oxygen-rich combustion on soot formation in laminar co-flow propane diffusion flames. J. Energy Inst. 2020, 93, 822-832. [CrossRef]

16. Chu, H.; Han, W.; Cao, W.; Tao, C.; Raza, M.; Chen, L. Experimental investigation of soot morphology and primary particle size along axial and radial direction of an ethylene diffusion flame via electron microscopy. J. Energy Inst. 2019, 93, 1294-1302. [CrossRef] 